\title{
A Comprehensive Orbit Reconstruction for the Galileo Prime Mission in the J2000 System
}

\author{
by \\ Robert A. Jacobson \\ Robert J. Haw \\ Tim P. McElrath \\ Peter G. Antreasian \\ Jet Propulsion Laboratory \\ California Institute of Technology \\ $\mathrm{M} / \mathrm{S} 301-150$ \\ 4800 Oak Grove Drive \\ Pasadena, CA 91109 \\ Phone: (818) 354-7201 \\ FAX: (818) 393-6388 \\ email: raj@murphy.jpl.nasa.gov
}

January 28, 1999

submitted to

AAS/AIAA Astrodynamics Specialist Conference

Girdwood, Alaska

August 16-19, 1999 
The Cialileo spacecraft arrived at Jupiter in December of 1995 to begin an orbital tour of the Jovian system. The objective of the tour was the up close study of the planet, its satellites, and its magnetosphere. The spacecraft completed its 11 orbit prime mission in November of 1997 having had 16 successful close encounters with the Galilean satellites (including two prior to Jupiter orbit insertion). Galileo continues to operate and will have made an additional 10 orbits of Jupiter by the date of this Conference. Earlier papers (Antreasian et al. 1997, Haw et al. 1997) discuss the determination of the spacecraft orbit in support of mission operations from arrival at Jupiter through the first 9 orbits. In this paper we re-examine those earlier orbits and extend the analysis through orbit 12 , the first orbit of the Galileo Europa Mission (GEM). Table 1 summarizes the geometry of the satellite encounters on each of those orbits. The objective of our work is the reconstruction of the spacecraft trajectory together with the development of a consistent set of ephemerides for the Galilean satellites. As a necessary byproduct of the reconstruction we determine improved values for the Jovian system gravitational parameters and for the Jupiter pole orientation angles. Our preliminary analyses have already led to many of the results reported in the scientific literature (Anderson et al. 1998a; Anderson et al. 1998b; Anderson et al. 1998c; Jacobson 1998).

Unlike the Galileo Navigation Team which operates in the EME-1950 coordinate system, we elected to work in the (J2000) International Celestial Reference Frame (ICRF), the reference frame of the current JPL planetary and satellite ephemerides as well as the standard frame of the international astronomical and planetary science community. Use of this frame permits more precise modelling of the spacecraft and satellite observations. Moreover, it is the frame of choice for all other operational JPL missions and will probably be the frame for future missions for some time. Consequently, our adoption of the ICRF will facilitate the combination of our results with any obtained from future missions (e.g. the proposed Europa Orbiter mission). In addition, our results may be used by the science community without need of a reference frame conversion.

To determine the orbits of the spacecraft and satellites we adjusted parameters in the dynamical model of their motions to obtain a fit to observations. The motion model includes gravitational dynamics (attractions of the satellites, Jupiter, the Sun, and other solar system planets) which affect both the spacecraft and satellites and non-gravitational dynamics (solar radiation pressure and thrusting maneuvers) which affect only the spacecraft. The fundamental adjustable parameters included:

- epoch position and velocity of the spacecraft and each Galilean satellite

- GM's of the planetary system and the Galilean satellites

- gravitational harmonics of the planet and Galilean satellites

- Jupiter pole orientation angles

- specular and diffuse reflectivities in the spacecraft solar radiation pressure model

- thrust magnitude and direction for large spacecraft maneuvers

- impulsive velocity changes for small spacecraft maneuvers 
The model was fit to the following observations:

- spacecraft Doppler tracking

- spacecraft very-long baseline interferometry (VLBI)

- spacecraft radio occultations."

- spacecraft optical navigation imaging

- satellite photometric Earthbased astrometry

- satellite CCD Earthbased astrometry

- satellite mutual events (mutual eclipses and occultations)

- satellite eclipse timings (eclipses by Jupiter)

- satellite positions from the Voyager spacecraft

The Galileo optical navigation data, originally referenced to EME-1950 system, were modified; the reference star locations were replaced with $\mathrm{J} 2000$ positions from the Hipparcos and Tycho catalogs (fortunately the stars appeared in at least one of these catalogs). The new positions are the best available J2000 positions of the stars. In order to obtain an adequate fit to the observations we also had to adjust a number of parameters in the observation model. These included:

- one-way Doppler bias and drift

- station dependent two-way and three-way Doppler biases

- day and night ionosphere delays

- spacecraft camera pointing

We processed the Galileo spacecraft data for each orbit separately, i.e., we determined an epoch state vector for each. The orbits for the most part extended from one Jupiter apoapsis to the next. In order to force a degree of continuity between the orbits, we imposed an equality constraint, in a least squares sense, on the spacecraft positions at the beginning and end times of each orbit. Orbit 0 (the Io encounter) is disjoint from the other orbits as we made no attempt to account for the Jupiter orbit insertion maneuver. The Io encounter is included because it provides significant information on Io's orbit and gravity field.

The paper provides a detailed discussion of our observation processing and its outcome. We examine the quality of the fit to the observations, and we give the values of the parameters in our reconstructed dynamical model and their uncertainties. Also we compare our reconstructed orbits to the orbits determined during mission operations and our gravitational parameters to those appearing in the scientific literature.

\section{Acknowledgement}

The research described in this paper was performed at the Jet Propulsion Laboratory, California Institute of Technology, under contract with the Vational Aeronautics and Space Administration. 


\section{References}

1. Anderson, J.D., Schubert, G., Jacobson, R.A., Lau, E.L., Moore, W.B., and Sjogren, W.L. (1998a) 'Distributiop of Rock, Metals, and Ices in Callisto', Science Vol. 280 pp. 1573-1576.

2. Anderson, J.D., Schuhert, G., Jacobson, R.A., Lau, E.L., Moore, W.B., and Sjogren, W.L. (1998b) 'Europa's Differentiated Internal Structure: Inferences from Four Galileo Encounters', Science Vol. 281 pp. 2019-2022.

3. Anderson, J.D., Jacobson, R.A., Lau, E.L., Sjogren, W.L., Schubert, G., Moore, W.B. (1998c) 'Interior Structure of the Galilean Satellites', 1998 Bull. AAS 30, No. 2,826

4. Antreasian, P.G., McElrath, T.P., Haw, R.J., \& Lewis, G.D. (1997) "Galileo Orbit Determination Results During the Satellite Tour", AAS Paper No. 97699, AAS/AIAA Astrodynamics Specialist Conference, Sun Valley, Idaho

5. Haw, R.J., Antreasian, P.G., Graat, E.G., McElrath, T.P., \& Nicholson, F.T. (1997) 'Navigating Galileo at Jupiter Arrival', AIAA Journal of Spacecraft and Rockets 34, No. 4, pp. 503.

6. Jacobson, R.A. (1998) 'The Orbits and Masses of the Galilean SatellitesResults from the Galileo Mission', 1998 Bull. AAS 30, No.3, 1147

Table 1: Encounter Geometry and Times

\begin{tabular}{|c|c|c|c|c|c|}
\hline Encounter & Orbit & Alt. & Lat. & Long. & Time (ET) \\
\hline Europa & 0 & 33012 & -64.21 & 75.86 & 07-Dec-1995 13:09:53.18 \\
\hline Io & 0 & 897 & -9.59 & 259.06 & 07-Dec-1995 17:46:59.54 \\
\hline Ganymede & 1 & 835 & 30.39 & 246.62 & 2 ז-Jun-1996 06:30:08.86 \\
\hline Ganymede & 2 & 261 & 79.29 & 236.32 & 06-Sep-1996 19:00:36.04 \\
\hline Callisto & 3 & 1136 & 13.20 & 282.25 & 04-Nov-1996 13:35:29.87 \\
\hline Europa & 3 & 34787 & 0.66 & 125.96 & 06-Nov-1996 18:50:53.36 \\
\hline Europa & 4 & 692 & $-1.6 \vec{\imath}$ & 322.46 & 19-Dec-1996 06:53:59.94 \\
\hline Europa & 6 & 586 & -17.02 & 34.61 & 20-Feb-1997 17:07:12.41 \\
\hline Europa & 7 & 23486 & 2.14 & 226.25 & 04-Apr-1997 05:59:49.80 \\
\hline Ganymede & 7 & 3102 & 55.79 & 270.37 & $05-$ Apr-1997 07:11:00.30 \\
\hline Callisto & 8 & 33060 & -42.00 & 287.72 & 06-May-1997 12:11:25.01 \\
\hline Ganymede & 8 & 1603 & 28.27 & 84.85 & 07 -May-1997 15:57:11.74 \\
\hline Callisto & 9 & 418 & 1.96 & 100.98 & $2.5-J u n-199-\quad 13: 48: 52.15$ \\
\hline Ganymede & 9 & 79740 & 0.04 & 261.23 & 26-Jun-1997 1 $7: 20: 36.52$ \\
\hline C'allisto & 10 & 535 & 4.60 & 281.29 & 17-Sep-199700:19:57.98 \\
\hline Europa & 11 & 2043 & 25.73 & 218.71 & 06-Nov-1997 $20: 32: 47.39$ \\
\hline Ganymede & 12 & $1+403$ & -.5 .81 & 266.14 & 15-Dec-1997 09:59:12.55 \\
\hline Europa & 12 & 201 & .8 .67 & $1: 3 \cdot 4.32$ & 16-Dec-199) $\quad$ :2:04:2:3.05 \\
\hline
\end{tabular}

